\title{
Voltammetric Determination of Acetaminophen in the Presence of Codeine and Ascorbic Acid at Layer-by-Layer MWCNT/Hydroquinone Sulfonic Acid-Overoxidized Polypyrrole Modified Glassy Carbon Electrode
}

\author{
Saeed Shahrokhian ${ }^{1,2}$ and Reyhaneh-Sadat Saberi ${ }^{1}$ \\ ${ }^{1}$ Department of Chemistry, Sharif University of Technology, Tehran 11155-9516, Iran \\ ${ }^{2}$ Institute for Nanoscience and Technology, Sharif University of Technology, Tehran 11155-9516, Iran \\ Correspondence should be addressed to Saeed Shahrokhian, shahrokhian@sharif.edu \\ Received 26 March 2011; Revised 19 May 2011; Accepted 21 May 2011 \\ Academic Editor: Farnoush Faridbod
}

Copyright () 2011 S. Shahrokhian and R.-S. Saberi. This is an open access article distributed under the Creative Commons Attribution License, which permits unrestricted use, distribution, and reproduction in any medium, provided the original work is properly cited.

\begin{abstract}
A very sensitive electrochemical sensor constructed of a glassy carbon electrode modified with a layer-by-layer MWCNT/dopedoveroxidized polypyrrole (oppy/MWCNT /GCE) was used for the determination of acetaminophen (AC) in the presence of codeine and ascorbic acid (AA). In comparison to the bare glassy carbon electrode, a considerable shift in the peak potential together with an increase in the peak current was observed for AC on the surface of oppy/MWCNT/GCE, which can be related to the enlarged microscopic surface area of the electrode. The effect of the experimental conditions on the electrode response, such as types of counter ion, pyrrole and counter ion concentration, potential and number of cycles in the polymerization procedure, amount of MWCNT, and the $\mathrm{pH}$, were investigated. Under the optimized conditions, the calibration curve was obtained over two concentration ranges of $2 \times 10^{-7}-6 \times 10^{-6} \mathrm{M}$ and $4 \times 10^{-5}-1 \times 10^{-4} \mathrm{M}$ of AC with a linear correlation coefficient $\left(R^{2}\right)$ of 0.9959 and 0.9947 , respectively. The estimated detection limit $(3 \sigma)$ for AC was obtained as $5 \times 10^{-8} \mathrm{M}$. The developed method was successfully applied to analyze the pharmaceutical preparations of AC, and a recovery of $95 \%$ with a relative standard deviation of $0.98 \%$ was obtained for AC.
\end{abstract}

\section{Introduction}

The remarkable application potential of conducting polymers (CP) in chemical and biological sensors is one of the main reasons for the intensive investigation and development of the studies on these materials [1]. In the case of polypyrrole (ppy), useful features considered include its capacity to form adhesive coatings at different substrates, the possibility of growing it in aqueous media, easiness for chemical substitution to modify its properties, high porosity that enables fast kinetics of ion exchange with the surrounding medium, high electronic conductivity, high chemical stability, thickness controllability, ease of electrochemical polymerization, and good reversibility between its conducting and insulating states. These characteristics make ppy suitable in various electrochemical determinations including voltammetric and potentiometric [2], amperometric [3], and impedimetric $[4,5]$ techniques for the determination of various molecular species.

Polypyrrole in its oxidized form is a positively charged conducting polymer. Upon the overoxidation process, it loses its conductivity and charge and instead, its porosity would be improved. Characterization of these films revealed that overoxidation results in addition of carbonyl and carboxyle groups to the structure of the conducting polymer. These groups attract cationic species and reciprocally reject negatively charged ones, for example, ascorbate anions [6].

Carbon nanotubes (CNT) are considered as a novel form of carbon materials in two past decades [7]. In the recent years, carbon nanotubes (CNTs) have also been incorporated into the electrochemical sensors. While they have many properties of the other types of carbon, they offer unique 
advantages including enhanced electronic properties, a large edge/basal plane ratio, and facile kinetics of the electrode processes. Therefore, in comparison to the traditional carbon electrodes, CNT-based sensors generally have higher sensitivities, lower limits of detection, and faster electron transfer kinetics [8].

Composites of intrinsically conducting polymer (ICP) are materials that utilize conjugated polymers with at least one secondary component that can be inorganic/organic materials or biologically active species. The goal is to produce a new composite material with distinct properties that were not observed in the individual components. This may include either new or improved chemical properties that can be exploited for chemical or biological sensing. Addition of carbon nanotubes as secondary component drastically influences the electrical and thermal conductivity of ICPs [9]. Sensor modification by this method is performed in two ways: layer by layer [10] and electropolymerization in a mixture of monomer and CNT $[11,12]$. These modifications have concentrated on improving the electrical conductivity, mechanical strength, or electrochemical capacitance of the polymer. These polymer/CNT modified electrodes have been used successfully in electrochemical sensing applications $[13,14]$.

Acetaminophen (paracetamol, N-acetyl-p-aminophenol, AC) is an extensively used antipyretic analgesic drug and a suitable alternative for patients who are sensitive to aspirin [15]. Generally, AC does not exhibit any harmful side effects, but hypersensitivity or overdoses are known to cause severe liver and kidney damages, and adverse effects include rashes, blood dyscrasias, and pancreatitis $[16,17]$. Therefore, acetaminophen quantity control in pharmaceutical formulation is vital since, as mentioned above, overdosage could be dangerous.

$\mathrm{AC}$ as an analgesics drug is often used in the presence of other drugs like; aspirin, cetirizine, tramadol, and codeine. So acetaminophen determination could be affected by the second ingredient in pharmaceutical formulation. Development of a method that could determine acetaminophen in pharmaceutical and clinical preparations without any interference from the other ingredient is of great importance. Since the electrochemical detection of AC has advantages including low cost, fast response, simple instrumentations, high sensitivity, and facile miniaturization, this kind of method has attracted a lot of attentions. Various electrodes such as poly(taurine)/MWCNT/GCE [18], nano$\mathrm{TiO}_{2}$ /polymer/GCE [19], polypyrrole modified glassy carbon electrode [20], multiwall carbon nanotube (MWCNT) modified basal plane pyrolytic graphite electrode [21], single-walled carbon nanotube-film coated electrodes [22], polyaniline/MWCNT composite modified electrodes [23], carbon film resistor electrodes [24], gold nanoparticle modified carbon paste electrode [25], and thionine-immobilized multiwalled carbon nanotube modified carbon paste electrode [26] have been used for the voltammetric detection of AC.

In the present work, an oppy-MWCNT modified electrode has been fabricated by a new counter ion, hydroquinone sulfonic acid (HQSA). Cyclic voltammetry was used to investigate the electrocatalytic oxidation of $\mathrm{AC}$ at oppy-MWCNT modified electrode. The results show a considerable increase in the corresponding peak current (up to 6-times) together with a decrease in the peak potential $(\sim 110 \mathrm{mV})$. Differential pulse voltammetry (DPV) was successfully used for the AC determinations in the presence of AA and codeine. Effects of pyrrole and counter ion concentration, potential, and number of cycles for polymerization, volume of the casted MWCNT suspension, and $\mathrm{pH}$ value of the test solution on the current response of the modified electrode toward AC were investigated and optimized. The prepared electrochemical sensor showed some remarkable advantages, for example, low detection limit, excellent reproducibility, and relatively wide linear dynamic range.

\section{Experimental}

2.1. Chemicals. Multi-wall carbon nanotubes (MWCNT, purity $>95 \%$ ) with outer diameter less than $10 \mathrm{~nm}$ and tubes length of 5-15 $\mu \mathrm{m}$ were prepared from Nanostructured \& Amorphous Materials (USA). Acetaminophen (AC) and ascorbic acid (AA) were from Merck. Codeine was taken kindly from Kharazmi drug Company. Hydroquinone sulfonic acid (HQSA) was purchased from Fluka and used as counter ion, in the electropolymerization procedure. Pyrrole and dimethylformamide (DMF) were purchased from Merck. Stock solutions of AC were freshly prepared as required in $0.1 \mathrm{M}$ of appropriate buffer solutions. In these experiments, $0.1 \mathrm{M}$ acetate was used for preparation of $\mathrm{pHs}$ 4 and 5, and 0.1 $\mathrm{M}$ phosphate for $\mathrm{pHs} 3,6$, and 7. Aqueous solutions were prepared using doubled distilled water, which is prepared over the alkaline dilute permanganate solution. All solutions were deaerated by purging with nitrogen gas (99.999\%) prior to each experiment. During the experiments, nitrogen gas was passed over the surface of the test solutions in order to avoid entrance of oxygen into the solution.

2.2. Apparatus. All electrochemical measurements were performed with a Metrohm Computrace Voltammetric Analyzer (model 757 VA) instrument. An electrochemical cell with a three-electrode configuration was used with a glassy carbon electrode (3 mm diameter, Princeton Applied Research, unmodified and/or modified) as working electrode. Counter and reference electrodes were a pt wire and saturated $\mathrm{Ag} / \mathrm{AgCl}$ electrode, respectively.

A digital pH/mV/Ion meter (Metrohm, $827 \mathrm{pH} \mathrm{lab)}$ was utilized for preparation of the buffer solutions, which were used as supporting electrolyte in the voltammetric experiments.

2.3. Preparation of the Modified Electrode. Besides the fact that conducting polymers exhibit interesting properties, the rate of their interconversion is usually slow, because of the slow transport of counter ions into the polymer layer to maintain the charge balance. It is found that nanotubular structures are good candidates for realizing rapid switching between redox states. For this purpose, the appropriate 
amount of pure MWCNT was functionalized under concentrated nitric acid treatment process for 24 hours in order to obtain more edge sites and better dispersion of nanotubes $[27,28]$. After functionalization procedure, $1 \mathrm{mg}$ of the functionalized MWCNT (CNT-COOH) was dispersed in $1 \mathrm{~mL}$ of DMF solvent under ultrasonic agitation for $1 \mathrm{~h}$ prior to use. Then a $15 \mu \mathrm{L}$ of the dispersed CNTs was taken with a microsyringe, and after casting on the electrode surface, the electrode was heated in oven at $50^{\circ} \mathrm{c}$ for $15 \mathrm{~min}$. The electrode was then kept in room temperature for 1 hour (named MWCNT/GC electrode). The electrodeposition of the ppy film on the surface of GC electrode precoated with CNT was carried out from an aqueous solution containing $0.02 \mathrm{M}$ HQSA and $0.02 \mathrm{M}$ pyrrole by potential cycling between $0.0 \mathrm{~V}$ and $+0.75 \mathrm{~V}$ (versus $\mathrm{Ag} / \mathrm{AgCl}$ ) at scan rates of $50 \mathrm{mVs}^{-1}$ for a total of five scans (Figure 1). Since the counter ion (HQSA) appears as an electroactive species, as seen in Figure 1, the corresponding redox peak currents are increased by progressing the electropolymerization procedure. The increase in the redox peak current via polymerization reveals more surface active area for the polymer-modified electrode. These advantages for HQSA make it as an interesting counter ion that affects various properties of the prepared conducting polymer in its applications as an electrochemical sensor. After the electropolymerization procedure, the electrode was overoxidized by potential cycling between $-0.2 \mathrm{~V}$ to $1.3 \mathrm{~V}$ (versus $\mathrm{Ag} / \mathrm{AgCl}$ ) at scan rate of $100 \mathrm{mVs}^{-1}$ for a total of thirty scans in buffer solution of $\mathrm{pH}$ 7.0. This electrode recognized as oppy/MWCNT/GCE in the following parts. On the other hand, in comparison to ppy films on the bare GC electrode formed under similar conditions, the polymer films on CNT were notably less brittle and more adhesive to the electrode surface.

\section{Results and Discussion}

3.1. AFM Studies on the Electrode Surface. It has been reported that the overoxidation process on ppy films caused the increase of the porosity of the polymer film and created negative sites via carboxylate groups formed in its structure [6]. In order to prove the effect of the overoxidation on the porosity of the ppy film, the AFM images were obtained for MWCNT/GCEs, coated with ppy and over oxidized ppy. As can be seen in Figure 2, the overoxidation creates some grooves and pores in polymer film, leading to increase of the microscopic area of the electrode and facilitating the interlayer diffusion of the analyte species. Therefore, it can be predicted that by using this modified electrode, higher sensitivities in the electrochemical responses can be obtained.

3.2. Cyclic Voltammetric Studies. The electrochemical behavior of $\mathrm{AC}$ was investigated by the means of cyclic voltammetry $(\mathrm{CV})$ technique on the surface of different electrodes including bare glassy carbon electrode (GC), glassy carbon electrode coated with CNT (MWCNT/GC), and layer-bylayer modified electrode (oppy/MWCNT/GCE), respectively. As can be seen in Figure 3, the electrooxidation of AC is very weak and totally irreversible on the surface of bare GC. On

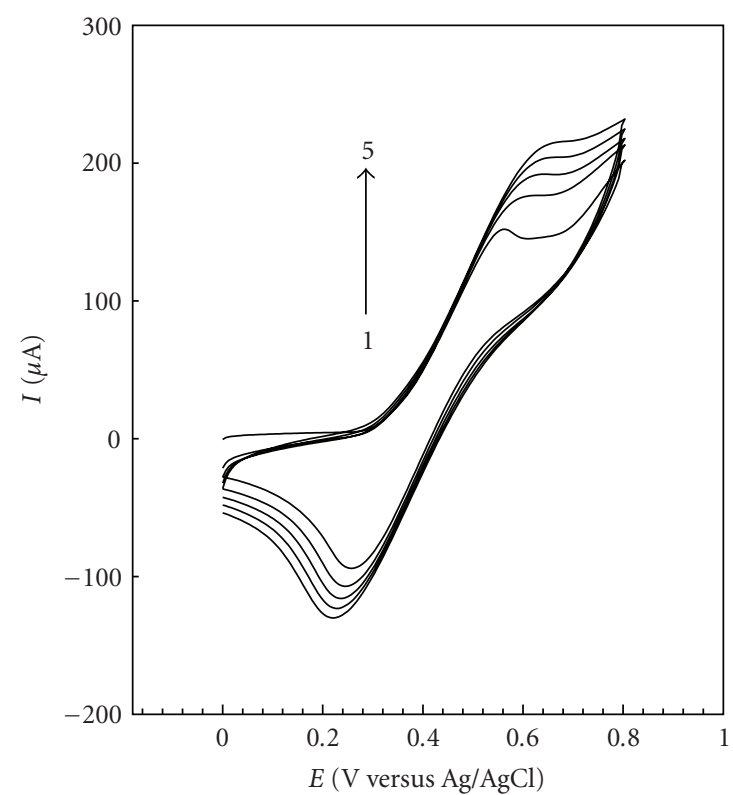

(a)

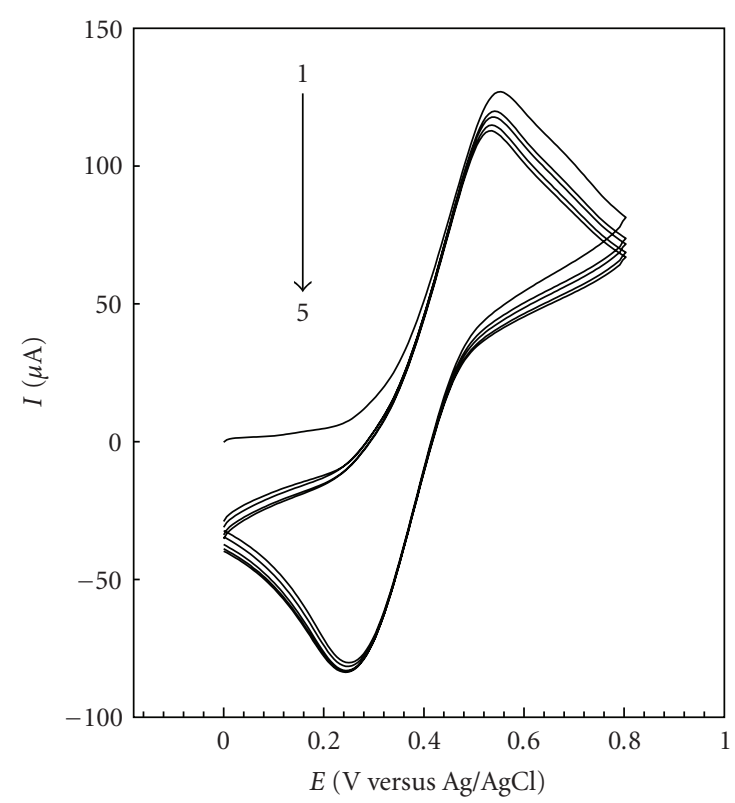

(b)

Figure 1: Consecutive cyclic voltammograms of (a) $0.02 \mathrm{M}$ pyrrole and $0.02 \mathrm{M}$ HQSA during electropolymerization process and (b) $0.02 \mathrm{M}$ HQSA.

the other hand, on the surface of the nanotube-modified electrode (MWCNT/GCE), the peak current is remarkably increased from about $11 \mu \mathrm{A}$ to $68 \mu \mathrm{A}$ (up to 6-times) associated with a significant negative shift in the anodic peak potential $(\sim 110 \mathrm{mV})$. The investigations showed that the anodic peak current for AC oxidation on the surface of the oppy/MWCNT/GCE is about 1.5-times greater than that at glassy carbon electrode coated with only a film of nanotube (MWCNT/GCE). It means that the electropolymerization, 


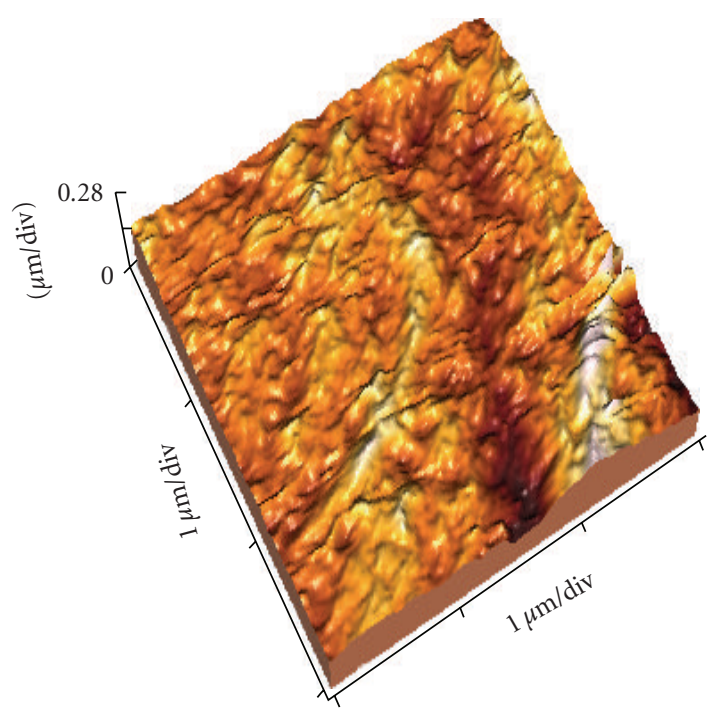

(a)

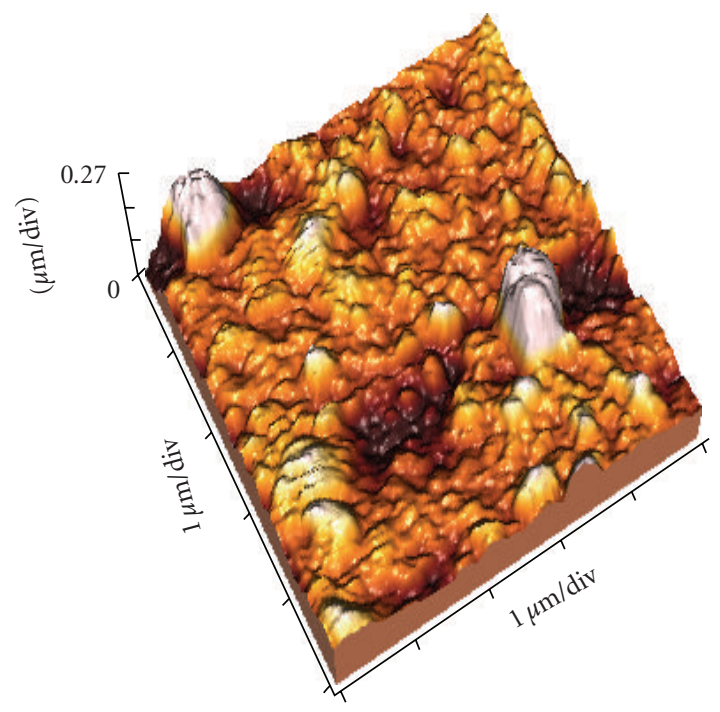

(b)

Figure 2: AFM image of (a) ppy/MWCNT/GCE and (b) oppy/ MWCNT/GCE.

and consequently overoxidation process, increased the active surface area of the electrode. On the other hand, by application of the oppy/MWCNT modified electrode the kinetics of the electron transfer for the electro-oxidation of AC is considerably improved (Figure 3 ). The catalytic activity of the modifier film caused a considerable decrease in the activation overpotential, increasing the sharpness of the waves and reversibility of the electrode process. It is obvious that the roughness characteristic of the electrode surface has a significant effect on the diffusion mechanism into the porous modifier film. The results of investigations showed that the overoxidized polymer on MWCNT/GCE has a more porous structure in relation to ordinary polymer on the surface of the MWCNT coated electrode. This improved

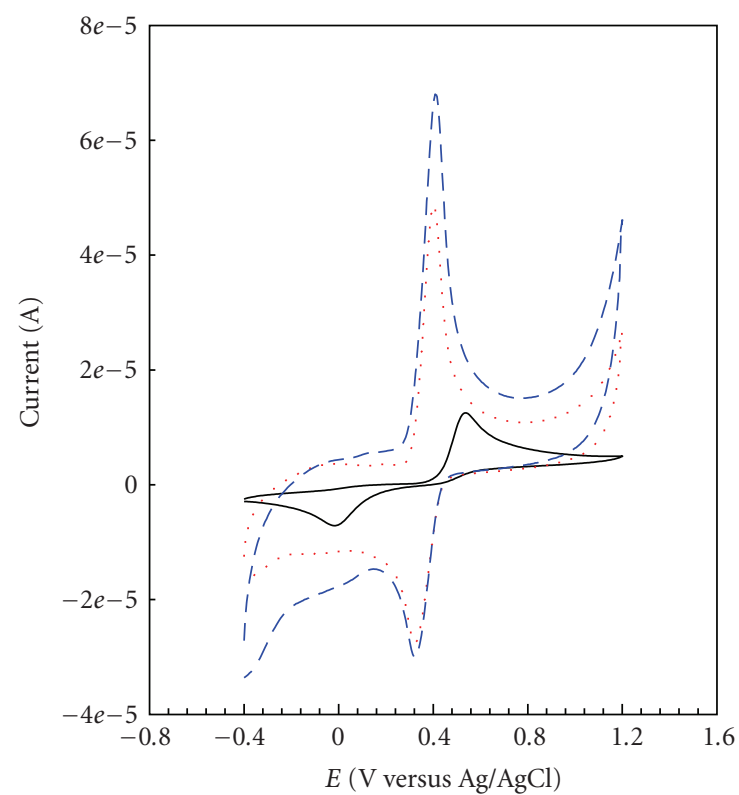

(a)

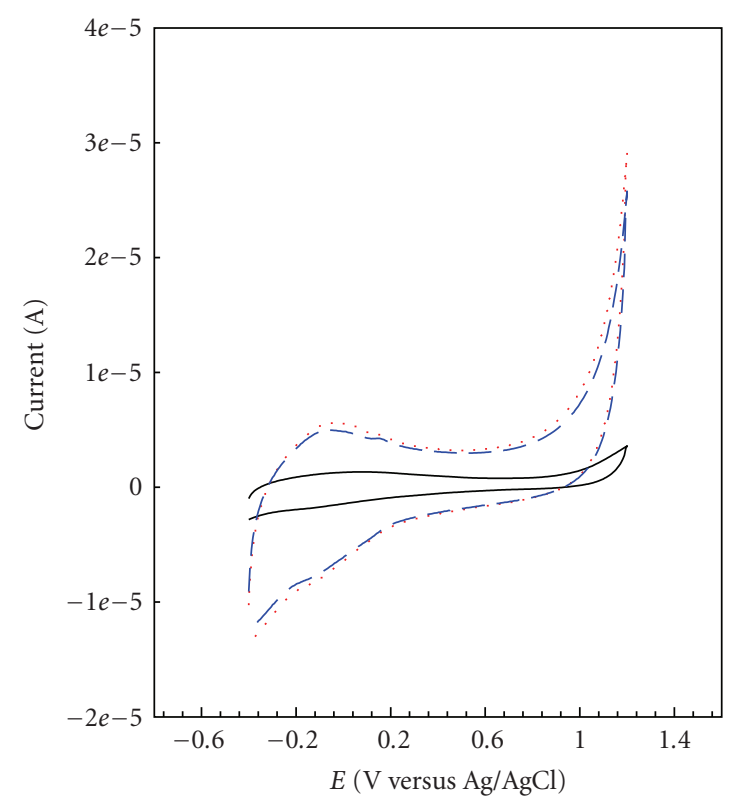

(b)

FIgUre 3: Cyclic voltammograms of various electrodes; bare GCE (-), CNT-GCE ( . . ) , and oppy/MWCNT/GCE (-- -). (a) $0.1 \mathrm{mM}$ $\mathrm{AC}$ and (b) buffer solution. Potential sweep rate was $100 \mathrm{mV} \mathrm{s}^{-1}$ and supporting electrolyte was $0.1 \mathrm{M}$ phosphate buffer solution of $\mathrm{pH}$ 7.0.

porosity enhances the diffusion of the analyte species via interlayer diffusion through the modifier thin film $[6,29$ 32]. A synergetic effect of the porosity of the overoxidized polymer/nanotube and great edge sites in the structure of functionalized MWCNT, which enhanced the catalytic effect of nanotube [29-32], resulted in very sharp and reversible voltammetric responses of AC. 
3.3. Effects of $p H$ and Potential Sweep Rate. In order to achieve the optimum $\mathrm{pH}$ for the anodic oxidation of $\mathrm{AC}$ on the surface of oppy/MWCNT/GCE, the electrochemical behavior was investigated in various $\mathrm{pHs}$ of the buffered solutions ( $\mathrm{pH}=3.0-7.0)$. From the results of cyclic voltammetric investigations (data not shown) the modified electrode shows electrochemical responses with very good sensitivity and repeatability in physiological conditions of $\mathrm{pH}$ 7.0. Since the more appropriate condition for analysis in biological samples is $\mathrm{pH} 7.0$, all experiments were performed in phosphate buffer solutions of this $\mathrm{pH}$ as supporting electrolyte. On the other hand, the negative shift in anodic peak potential $\left(E_{p, a}\right)$ with $\mathrm{pH}$ can be described by the following equation:

$$
E_{p, a}=+0.8164-0.057 \mathrm{pH} \quad\left(\mathrm{V} \text { versus } \frac{\mathrm{Ag}}{\mathrm{AgCl}}\right) .
$$

An experimental slope of $-57 \mathrm{mV} / \mathrm{pH}$ was obtained in these experiments that is close to the theoretical slope $(-59 \mathrm{mV} / \mathrm{pH})$ for a classical Nernstian two-electron, twoproton process. The difference between theoretical and experimental slopes can be related to the quasireversible mechanism.

The effect of potential sweep rate was also investigated in a solution containing $\mathrm{AC}$ with $\mathrm{pH}$ 7.0. Obviously, the oxidation process is controlled by adsorption as deduced from the linear dependence of the anodic peak current $\left(I_{p, a}\right)$ on the potential scan rate $(v)$ over a wide range of potential scan rates (from 5 to $400 \mathrm{mV} \mathrm{s}^{-1}$, data not shown).

3.4. Effect of the Thickness of Film Modifier. In order to investigate the effect of film thickness on the electrochemical responses toward $\mathrm{AC}$, the parameters influencing the film thickness including monomer and counter ion concentration, number of cycles during the electropolymerization, and the volume of CNT suspension were investigated. The optimum results (maximum peak currents for AC) were obtained using following conditions: $15 \mu \mathrm{L}$ of CNT suspension, polymerization solution containing $0.02 \mathrm{M}$ of pyrrole and $0.02 \mathrm{M} \mathrm{HSQA}$, and 7 consecutive cycles of potential in the range of 0.00 to $0.75 \mathrm{~V}$. The results showed that the type of counter ion has a very important role in the stability and conductivity of the polymer. In this regard, the electrochemical responses of the modified electrode incorporating HSQA were compared with the electrode containing $\mathrm{Cl}^{-}$as an inorganic counter ion (Figure 4). The response for polymer doped with HQSA is 2.5-times larger than polymer doped with $\mathrm{KCl}$. High stability of HQSA on the surface of the electrode can be related to its aromatic structure and effective $\pi-\pi$ interactions with polymer. It can be predicted that HQSA as an aromatic anion with 1 negative charge produced a polymer with better conductivity. It was found that the electrochemical responses of $\mathrm{AC}$ on the surface of oppy/MWCNT/GCE are decreased as the thickness of the film increased. On the other hand, by increasing the thickness of the polymer film, the capacitive background current is increased, which caused higher detection limit in voltammetric measurements. From these observations, it can

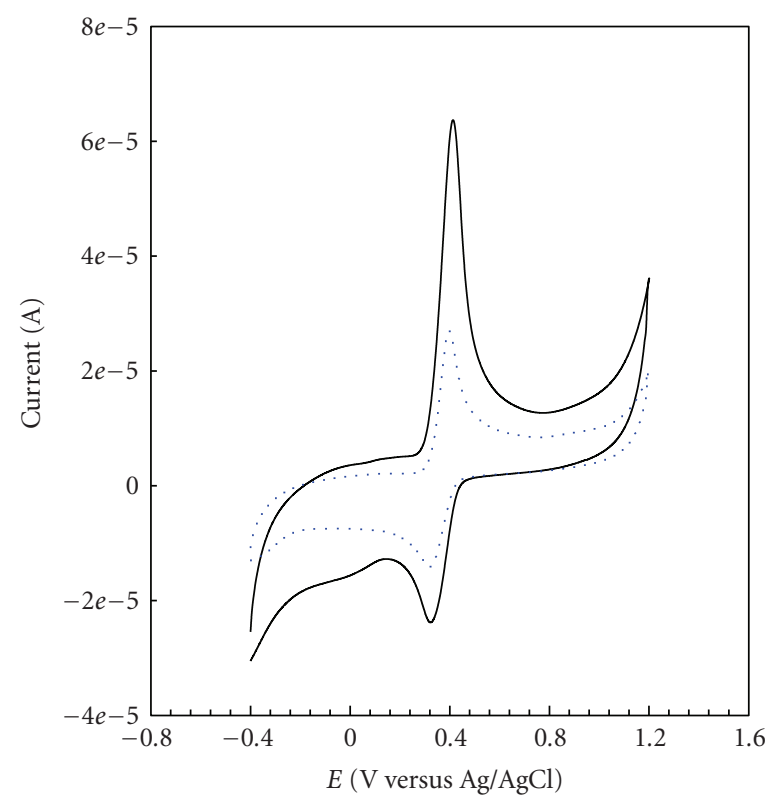

FIGURE 4: Cyclic voltammograms of $0.1 \mathrm{mM}$ AC on the surface of oppy/MWCNT/GCE in $\mathrm{pH}=7$ various polymerization counter ion; $\mathrm{KCl}$ (dotted line), and HQSA (solid line).

be concluded that the polymer layer with high thickness, which was formed on the surface of the CNT-coated GC electrode, makes the electrode surface passive and caused to some extent weakening the voltammetric responses. Our investigations with GCE coated with various amounts of CNT ( $\mu \mathrm{L}$ of suspension casted on the electrode surface) showed that the maximum sensitivity for the response toward AC can be obtained with $15 \mu \mathrm{L}$ of CNT suspension. A higher volume of suspension is caused to some inappropriate mechanical properties and decreased the adherence of the CNT film on the GCE surface. Therefore, a polymer-coated electrode with the above-mentioned optimum condition for the electropolymerization was chosen as the modified electrode in all voltammetric studies.

3.5. Voltammetric Response toward Ascorbic Acid. In the electrochemical investigations of AC in clinical preparations, presence of some potentially interfering compounds, especially ascorbic acid (AA), is considered as a significant problem in the accuracy of the determinations. This compound generally shows overlapping signals on the surface of most chemically modified electrodes, which limit the analytical applicability of the sensors.

In order to investigate the effect of $\mathrm{AA}$ on the response of the modified electrode, the cyclic voltammograms were recorded at various electrodes in buffer solutions of $\mathrm{pH}$ 7.0. As can be seen in Figure 5, a totally irreversible voltammetric response is resulted on the surface of bare GCE with a peak potential of $312 \mathrm{mV}$. On the surface of GCE coated with MWCNT, this peak is shifted to slightly less anodic potentials and the corresponding peak current is considerably increased. Based on this evident, AA must be considered as a serious interference in the determination 


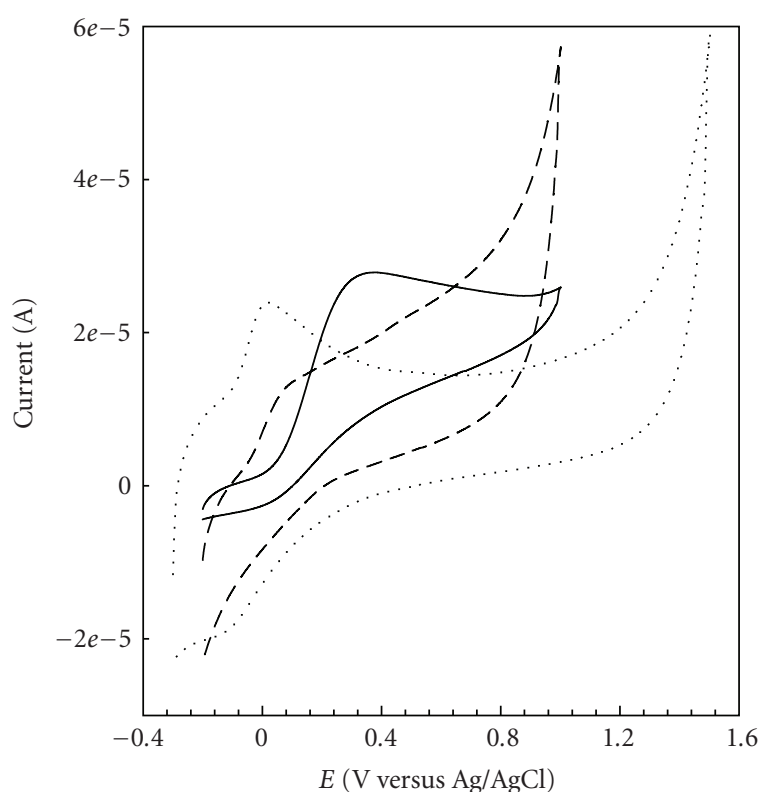

FIGURE 5: Cyclic voltammograms of $1 \mathrm{mM}$ AA in phosphate buffer solution of $\mathrm{pH} 7.0$ on the surface of various electrodes; bare GCE (solid line), CNT-GCE (dotted line), and oppy/MWCNT/GCE (dashed line). Potential sweep rate was $100 \mathrm{mV} \mathrm{s}^{-1}$.

of AC using these electrodes. On the other hand, on the surface of the oppy/MWCNT/GCE the voltammetric response of AA completely disappeared. Such a behavior was previously reported on the surface of the GCE coated with ppy doped with multivalent organic anions such as congo-red in determination of dopamine [33] and tiron in determination of uric acid [9] in solutions with $\mathrm{pH} \geq 5.0$. It is clear that AA with a $\mathrm{p} K_{a}$ of 4.17 can exist mainly as anionic form in the experimental conditions $(\mathrm{pH} 7.0)$ and therefore, has repulsive interaction with the anionic sites of the film-modified electrode. On the other hand, the presence of the carboxyl groups in the structure of the overoxidized polymer film, in addition to the presence of the HQSA anion, creates highly negative charge density that exclude anionic analyte species, like AA [6]. The induced charge discrimination by the modifier film makes the electrode suitable for simultaneous determination of $\mathrm{AC}$ in the presence of high concentrations of AA.

3.6. Analytical Characterization. Under the optimized conditions, the DPV of various concentrations of $\mathrm{AC}$ were recorded in buffered solutions of $\mathrm{pH} 7.0$ using an optimized accumulation time of $120 \mathrm{~s}$ (Figure 6). The calibration curve of the anodic peak current for solutions containing different amounts of AC is constructed and two linear ranges of $2 \times$ $10^{-7}-6 \times 10^{-6} \mathrm{M}$ and $4 \times 10^{-5}-1 \times 10^{-4} \mathrm{M}$ are obtained with correlation coefficients $\left(\mathrm{R}^{2}\right)$ of 0.9959 and 0.9947 and slopes of $22.01 \mu \mathrm{A} / \mu \mathrm{M}$ and $0.7257 \mu \mathrm{A} / \mu \mathrm{M}$, respectively. The reason for changing the slope is creation of a monolayer of accumulated AC at the surface of modified electrode, so for more accumulation on the surface of this new surface the slope of electrode will change. In these measurements, based

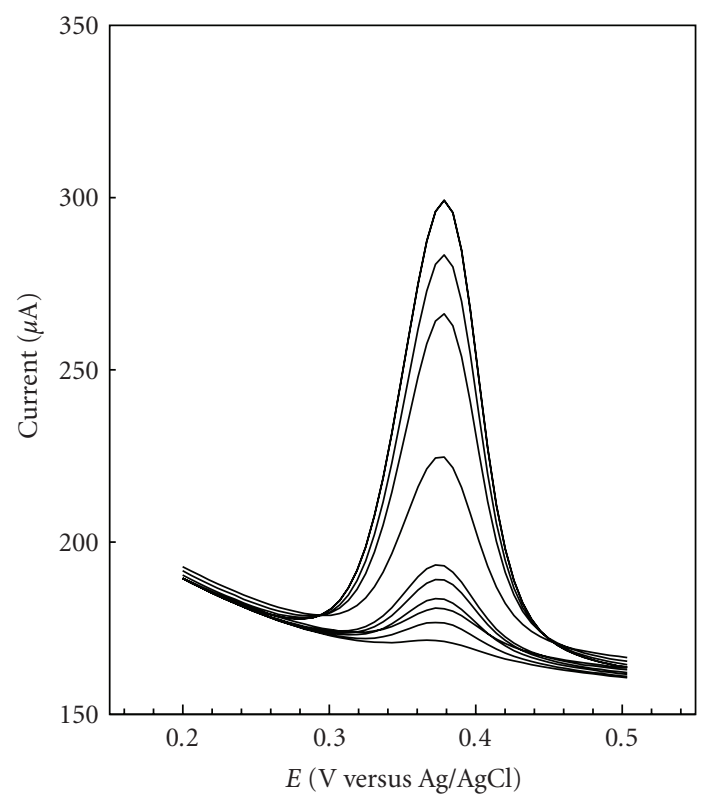

(a)

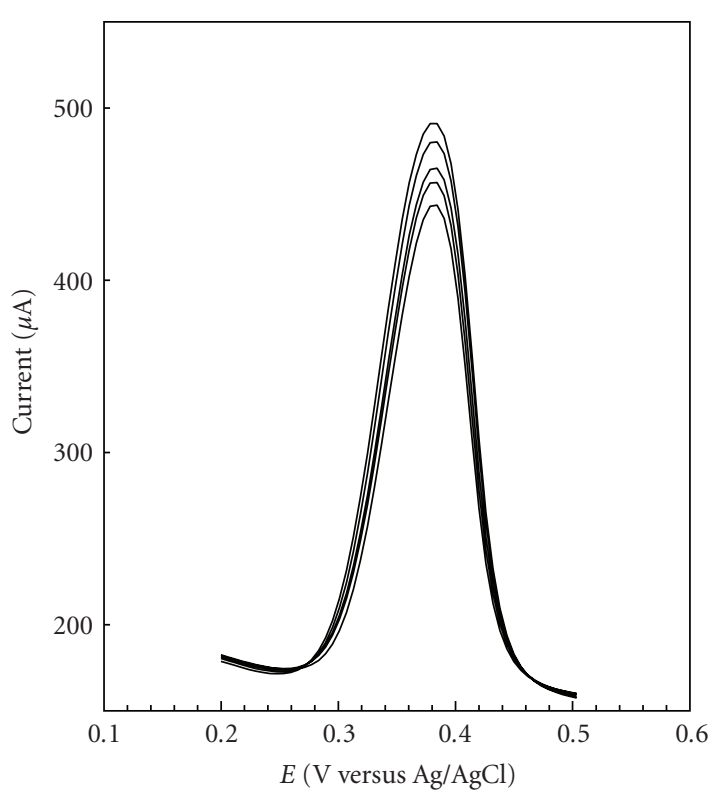

(b)

Figure 6: Differential pulse voltammograms of $0.1 \mathrm{M}$ phosphate buffer solution containing various concentrations of $\mathrm{AC}$ (down to up); (a) 0.2, 0.4, 0.5, 0.6, 0.8, 1, 2, 4, 5, and 6 and (b) 40, 50, 60, 80, and $100 \mu \mathrm{M}$ AC. Pulse amplitude was $50 \mathrm{mV}$. Accumulation time was $120 \mathrm{~s}$.

upon linear extrapolation of the first range of the calibration curve [34], a theoretical detection limit $(\mathrm{S} / \mathrm{N}=3)$ of $5 \times$ $10^{-8} \mathrm{M}$ is resulted for the voltammetric determination of AC.

$\mathrm{AC}$, as an analgesics drug, often used in the presence of other drugs like, aspirin, cetirzine, tramadol, and codeine. Results of the present work showed that none of these pharmaceuticals interfere with AC signal on the surface of the prepared modified electrode (Figure 7). In this work cyclic 


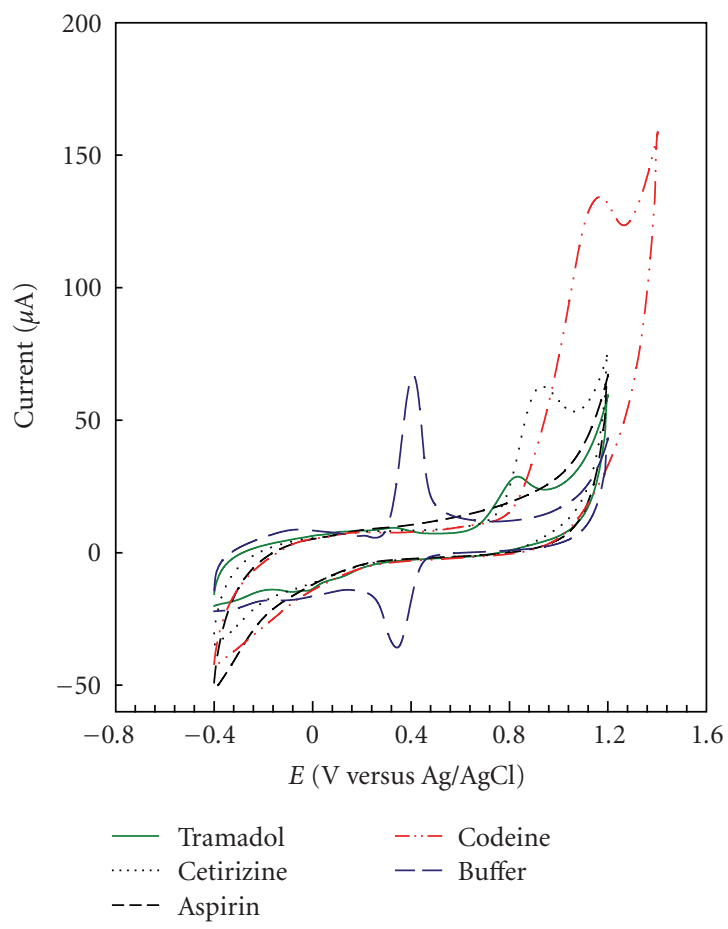

(a)

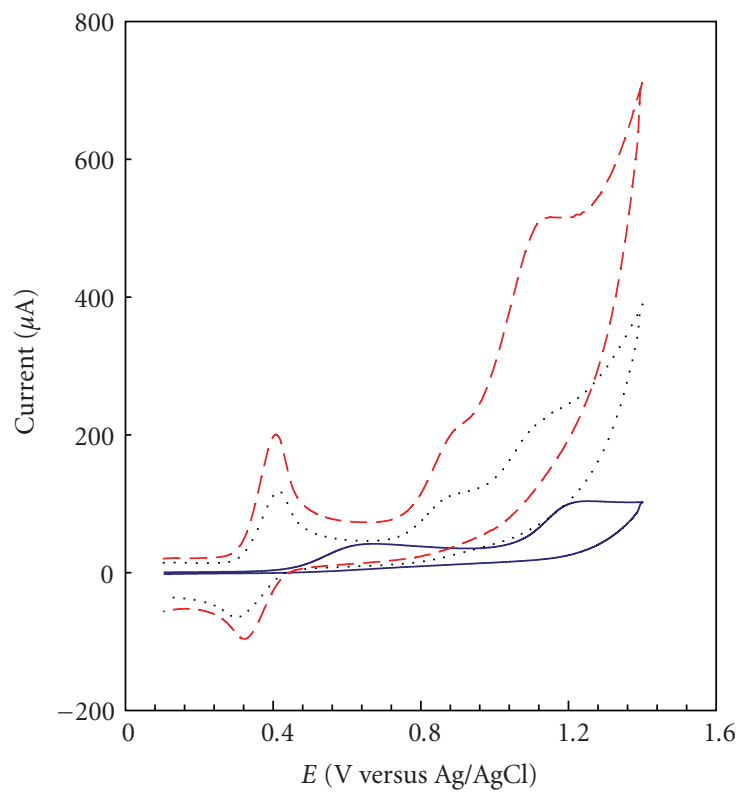

(b)

Figure 7: Cyclic voltammograms of (a) $0.1 \mathrm{mM}$ of several drugs: tramadol, cetirzine, aspirin, codeine, and AC on the surface of oppy/MWCNT/GCE, (b) mixture solution containing $1 \mathrm{mM}$ of AA, AC, and codeine on the surface of bare GCE (solid line), CNT-GCE (dotted line), and oppy/MWCNT/GCE (dashed line). Supporting electrolyte was phosphate buffer solution of pH 7.0 and potential sweep rate was $100 \mathrm{mV} \mathrm{s}^{-1}$.

voltammograms of $\mathrm{AC}$ in the presence of codeine and $\mathrm{AA}$ are compared on the surface of three different electrodes: bare GCE, CNT-GCE, and oppy/MWCNT/GCE. As can be seen in Figure 7(b), remarkable enhancements in the peak current and reversibility of $\mathrm{AC}$ is resulted on the surface of the modified electrode. Also, a complete resolution of its peak from codeine signal and masking the effect of AA response is obtained by applying the oppy/MWCNT modified electrode. The results represent the applicability of the prepared modified electrode for AC determinations with good accuracy in the presence of other common pharmaceuticals.

The DPV of various concentrations of AC in the presence of a constant amount of codeine $\left(1 \times 10^{-4} \mathrm{M}\right)$ are recorded in buffered solutions of $\mathrm{pH} 7.0$ in the optimized accumulation time (120 s, Figure 8(a)). The calibration curve of the anodic peak current versus the concentration of AC (in the presence of a constant amount of codeine) showed a linear behavior in the range of $2 \times 10^{-7}$ to $5 \times 10^{-6} \mathrm{M}$ with a correlation coefficient $\left(\mathrm{R}^{2}\right)$ of 0.994 and a slope of $5.1458 \mu \mathrm{A} / \mu \mathrm{M}$. Since AC and codeine both adsorb on the surface of the electrode, the competition for the adsorption caused to decrease the response sensitivity toward AC. The investigations showed that the sensitivity of electrode response toward AC decreased with a factor of 5 in the presence of codeine. However, the determination method still has a good detection limit about $6 \times 10^{-8} \mathrm{M}$ that makes the modified electrode a very good sensor for AC determinations in the pharmaceutical preparations.

Standard addition is performed by spiking various concentrations of $\mathrm{AC}$ in the range of 0.2 to $3 \mu \mathrm{M}$ to a fixed amount tablet sample. The calibration curve is obtained by plotting the peak current versus the spiked concentrations of AC. The resulted plot is compared with the plot obtained by addition of same concentrations of AC and constant amount of codeine to the buffer solution (Figure $8(\mathrm{~b})$ ). The results represent a very good accuracy for the determination of AC using the modified electrode. It means that the tablet matrix would not interfere in the voltammetric determination of AC. The results of the determinations in the presence of codeine (in the concentration range used in medical applications) represent a good recovery more than $95 \%$ for AC.

One of the remarkable advantages of the presented method is its low detection limit, which is obtained as $0.05 \mu \mathrm{M}$ for AC. The prepared electrode showed highly sensitive responses toward $\mathrm{AC}$ in solutions with a $\mathrm{pH}$ near to physiological conditions ( $\mathrm{pH}$ 7). Moreover, the prepared modified electrode showed very stable responses leading to a very good reproducibility that makes it a very sensitive and selective tool in determination of trace amounts of AC in pharmaceutical preparations. In this regard, Figure 9 shows the replicates DPVs during a day (a) and repetitive DPV measurements during 4 days (b) for $0.5 \mu \mathrm{M}$ AC. 


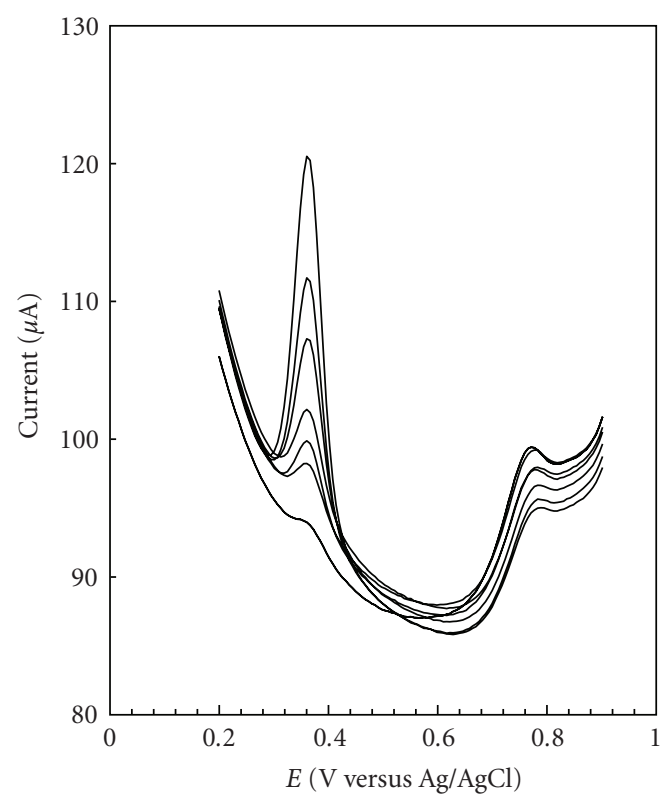

(a)

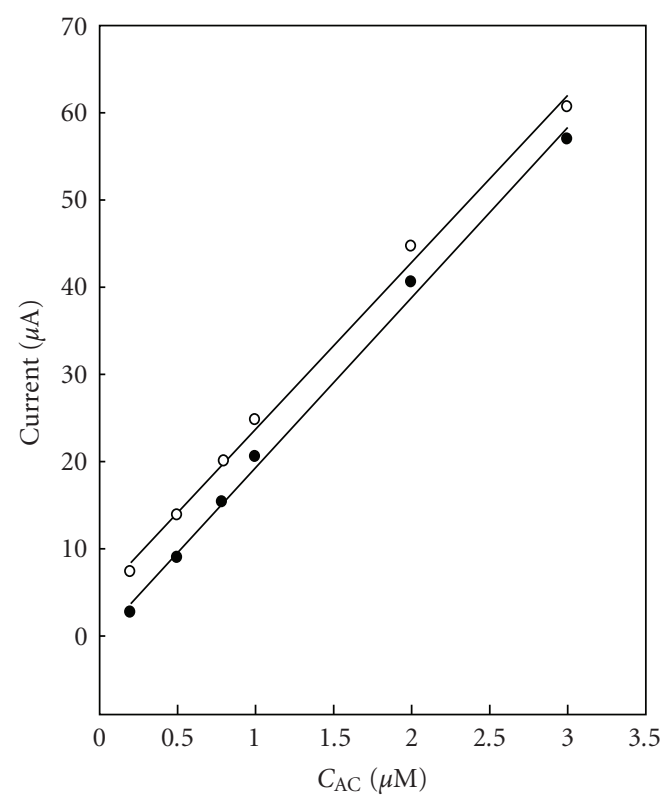

(b)

FIGURE 8: (a) Differential pulse voltammograms of $0.1 \mathrm{M}$ phosphate buffer solution containing various concentrations of AC (down to up); $0.2,0.5,0.8,1,2,3,5 \mu \mathrm{M}$ AC in the presence of $1 \times$ $10^{-4} \mathrm{M}$ codeine. (b) Linear calibration curve of the anodic peak current versus AC concentration added to $(\bullet) 0.1 \mathrm{M}$ phosphate buffer solution of pH 7.0 and constant amount of codeine, (o) AC tablet solution buffered in same $\mathrm{pH}$.

Comparison with the analytical characteristics of the previous reports for AC determinations showed that the prepared modified electrode in the present work has a good detection limit with a relatively wide linear dynamic range. In the other hand, most of the sensors have been used for the determination of AC alone. However, the prepared modified

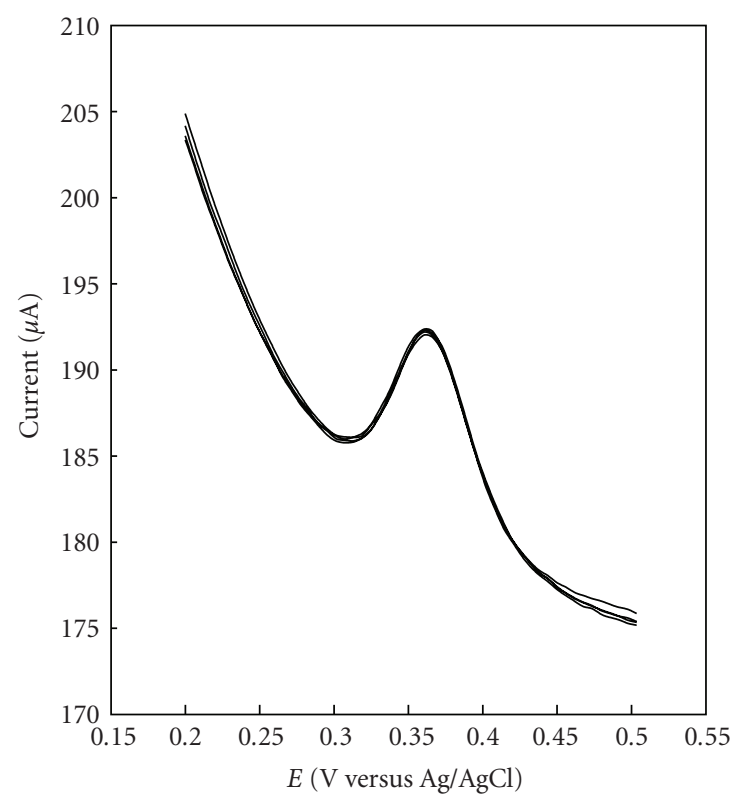

(a)

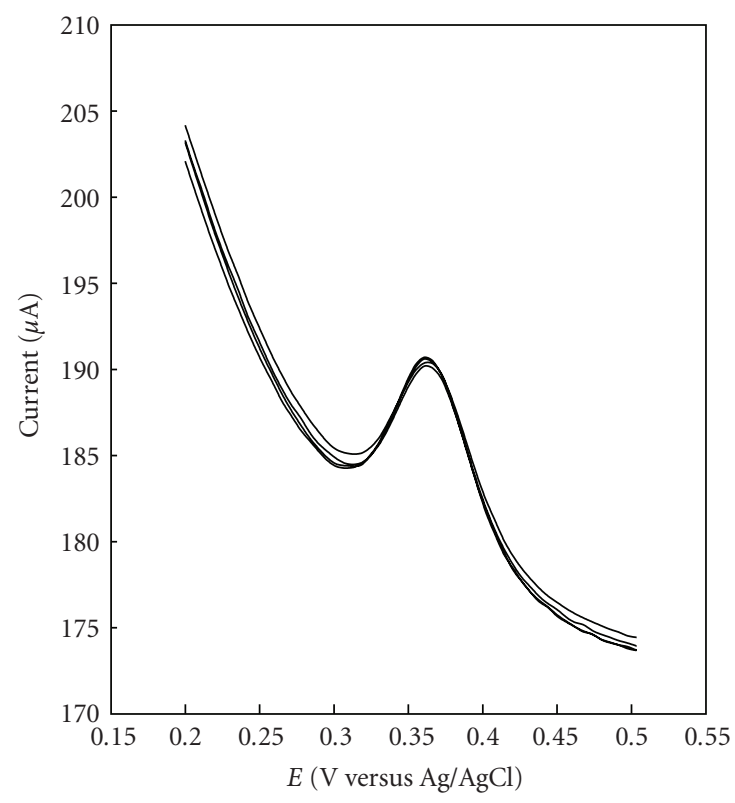

(b)

FIGURE 9: Four repetitive DPVs of $0.5 \mu \mathrm{M}$ AC on the surface of oppy/MWCNT/GCE during (a) one day and (b) four days. Potential sweep rate was $100 \mathrm{mV} \mathrm{s}^{-1}$ and supporting electrolyte was $0.1 \mathrm{M}$ phosphate buffer solution of $\mathrm{pH}$ 7.0.

electrode in this work can be successfully applied for the determination of $\mathrm{AC}$ in the presence of codeine with a low detection limit. Also, our investigations showed that other analgesics such as cetirizine, tramadol, and aspirin did not interfere in the voltammetric determinations of AC using the prepared modified electrode (Table 1). 
TABLE 1: Voltammetric response characteristics of the oppy/MWCNT/GCE toward AC in comparison to other reported electrodes.

\begin{tabular}{|c|c|c|c|c|}
\hline Electrode & Linear range $(\mu \mathrm{M})$ & Detection limit $(\mu \mathrm{M})$ & In the presence of other analytes & reference \\
\hline Gold nanoparticle modified CPE & $0.6-500$ & 0.3 & Alone & {$[25]$} \\
\hline Nanopolypyrrole modified GCE & $0.3-1.6$ & 0.25 & Alone & [20] \\
\hline Poly(taurine)/MWCNT/GCE & $1-100$ & 0.5 & Alone & [18] \\
\hline Nano- $\mathrm{TiO}_{2} /$ polymer/GCE & $12-120$ & 2 & Dopamine & [19] \\
\hline MWCNT/basal plane PGE & $0.01-2$ and $2-20$ & 0.01 & Alone & [21] \\
\hline Carbon film resistor & $0.8-500$ & 0.1 & Alone & {$[24]$} \\
\hline SWCNT/dicetyl phosphate film /GCE & $0.1-20$ & 0.04 & Alone & {$[22]$} \\
\hline PANI-MWCNT composite/GCE & $1-100$ and $250-2000$ & 0.25 & Alone & [23] \\
\hline Hematoxylin biosensor & $12-59$ and $59-262$ & NR & Noradrenaline & {$[26]$} \\
\hline Thionine MWCNT & $0.1-100$ & 0.05 & AA and Izoniazid & [27] \\
\hline oppy/MWCNT/GCE & $0.1-100$ & 0.05 & AA and codeine & This work \\
\hline
\end{tabular}

\section{Conclusions}

In this study, we have first introduced a new kind of counter ion for pyrrole polymerization that its redox behavior can show the surface enhancement during polymerization process. In the other hand, we present a good sensor with high sensitivity and quality for $\mathrm{AC}$ determination in the presence of codeine and ascorbic acid. This sensor shows an electrocatalytic behavior which leads to a significant increase in the peak current (11 to $68 \mu \mathrm{A}$ ) and a great decrease in the peak potential $(\sim 110 \mathrm{mV})$. All these observations, as well as other properties, such as excellent reproducibility, low detection limit, and charge repulsion toward AA on overoxidized polypyrrole film, make the modified electrode very useful in construction of a simple device for the determination of AC in pharmaceutical preparations.

\section{Acknowledgment}

The authors gratefully acknowledge the support to this paper by the Research Council and the Center of Excellence for Nanostructures of the Sharif University of Technology, Tehran, Iran.

\section{References}

[1] U. Lange, N. V. Roznyatovskaya, and V. M. Mirsky, "Conducting polymers in chemical sensors and arrays," Analytica Chimica Acta, vol. 614, no. 1, pp. 1-26, 2008.

[2] A. R. Zanganeh and M. K. Amini, "Polypyrrole-modified electrodes with induced recognition sites for potentiometric and voltammetric detection of copper(II) ion," Sensors and Actuators, B, vol. 135, no. 1, pp. 358-365, 2008.

[3] H. Peng, L. Zhang, C. Soeller, and J. Travas-Sejdic, "Conducting polymers for electrochemical DNA sensing," Biomaterials, vol. 30, no. 11, pp. 2132-2148, 2009.

[4] M. Singh, P. K. Kathuroju, and N. Jampana, "Polypyrrole based amperometric glucose biosensors," Sensors and Actuators, B, vol. 143, no. 1, pp. 430-443, 2009.

[5] A. Ramanavičius, A. Ramanavičienè, and A. Malinauskas, "Electrochemical sensors based on conducting polymerpolypyrrole," Electrochimica Acta, vol. 51, pp. 6025-6037, 2006.
[6] S. Iijima, "Helical microtubules of graphitic carbon," Nature, vol. 354, no. 6348, pp. 56-58, 1991.

[7] C. B. Jacobs, M. J. Peairs, and B. J. Venton, "Review: carbon nanotube based electrochemical sensors for biomolecules," Analytica Chimica Acta, vol. 662, no. 2, pp. 105-127, 2010.

[8] D. W. Hatchett and M. Josowicz, "Composites of intrinsically conducting polymers as sensing nanomaterials," Chemical Reviews, vol. 108, no. 2, pp. 746-769, 2008.

[9] S. Shahrokhian and E. Asadian, "Electrochemical determination of 1-dopa in the presence of ascorbic acid on the surface of the glassy carbon electrode modified by a bilayer of multiwalled carbon nanotube and poly-pyrrole doped with tiron," Journal of Electroanalytical Chemistry, vol. 636, no. 1-2, pp. 4046, 2009.

[10] Y. Li, P. Wang, L. Wang, and X. Lin, “Overoxidized polypyrrole film directed single-walled carbon nanotubes immobilization on glassy carbon electrode and its sensing applications," Biosensors and Bioelectronics, vol. 22, no. 12, pp. 3120-3125, 2007.

[11] S. Korkut, B. Keskinler, and E. Erhan, "An amperometric biosensor based on multiwalled carbon nanotubepoly(pyrrole)-horseradish peroxidase nanobiocomposite film for determination of phenol derivatives," Talanta, vol. 76, no. 5, pp. 1147-1152, 2008.

[12] G. Cheng, J. Zhao, Y. Tu, P. He, and Y. Fang, "A sensitive DNA electrochemical biosensor based on magnetite with a glassy carbon electrode modified by muti-walled carbon nanotubes in polypyrrole," Analytica Chimica Acta, vol. 533, no. 1, pp. 11-16, 2005.

[13] A. Zhang, J. Chen, D. Niu, G. G. Wallace, and J. Lu, "Electrochemical polymerization of pyrrole in $\mathrm{BMIMPF}_{6}$ ionic liquid and its electrochemical response to dopamine in the presence of ascorbic acid," Synthetic Metals, vol. 159, no. 15-16, pp. 1542-1545, 2009.

[14] W. Martindale and A. Wade, The Extra Pharmacopoeia, The Pharmaceutical Press, London, UK, 27th edition, 1979.

[15] B. D. Clayton and Y. N. Stock, Basic Pharmacology for Nurses, Mosby Inc, Harcourt Health Sciences Company, St. Louis, Mo, USA, 2001.

[16] J. E. F. Reynolds and W. Martindale, The Extra Pharmacopoeia, The Pharmaceutical Press, London, UK, 30th edition, 1993.

[17] Q. Wan, X. Wang, F. Yu, X. Wang, and N. Yang, "Poly(taurine)/ MWNT-modified glassy carbon electrodes for the detection of 
acetaminophen," Journal of Applied Electrochemistry, vol. 39, no. 6, pp. 785-790, 2009.

[18] S. A. Kumar, C. F. Tang, and S. M. Chen, "Electroanalytical determination of acetaminophen using nano- $\mathrm{TiO}_{2} /$ polymer coated electrode in the presence of dopamine," Talanta, vol. 76, no. 5, pp. 997-1005, 2008.

[19] B. Muralidharan, G. Gopu, C. Vedhi, and P. Manisankar, "Determination of analgesics in pharmaceutical formulations and urine samples using nano polypyrrole modified glassy carbon electrode," Journal of Applied Electrochemistry, vol. 39, no. 8, pp. 1177-1184, 2009.

[20] R. T. Kachoosangi, G. G. Wildgoose, and R. G. Compton, "Sensitive adsorptive stripping voltammetric determination of paracetamol at multiwalled carbon nanotube modified basal plane pyrolytic graphite electrode," Analytica Chimica Acta, vol. 618, no. 1, pp. 54-60, 2008.

[21] D. Sun and H. Zhang, "Electrochemical determination of acetaminophen using a glassy carbon electrode coated with a single-wall carbon nanotube-dicetyl phosphate film," Microchimica Acta, vol. 158, no. 1-2, pp. 131-136, 2007.

[22] M. Li and L. Jing, "Electrochemical behavior of acetaminophen and its detection on the PANI-MWCNTs composite modified electrode," Electrochimica Acta, vol. 52, no. 9, pp. 3250-3257, 2007.

[23] F. S. Felix, C. M. A. Brett, and L. Angnes, "Carbon film resistor electrode for amperometric determination of acetaminophen in pharmaceutical formulations," Journal of Pharmaceutical and Biomedical Analysis, vol. 43, no. 5, pp. 1622-1627, 2007.

[24] Z. Xu, Q. Yue, Z. Zhuang, and D. Xiao, "Flow injection amperometric determination of acetaminophen at a gold nanoparticle modified carbon paste electrode," Microchimica Acta, vol. 164, no. 3-4, pp. 387-393, 2009.

[25] N. Nasirizadeh and H. R. Zare, "Differential pulse voltammetric simultaneous determination of noradrenalin and acetaminophen using a hematoxylin biosensor," Talanta, vol. 80, no. 2, pp. 656-663, 2009.

[26] S. Shahrokhian and E. Asadian, "Simultaneous voltammetric determination of ascorbic acid, acetaminophen and isoniazid using thionine immobilized multi-walled carbon nanotube modified carbon paste electrode," Electrochimica Acta, vol. 55, no. 3, pp. 666-672, 2010.

[27] K. Esumi, M. Ishigami, A. Nakajima, K. Sawada, and H. Honda, "Chemical treatment of carbon nanotubes," Carbon, vol. 34, no. 2, pp. 279-281, 1996.

[28] A. Witkowski and A. Brajter-Toth, "Overoxidized polypyrrole films: a model for the design of permselective electrodes," Analytical Chemistry, vol. 64, no. 6, pp. 635-641, 1992.

[29] I. Streeter, G. G. Wildgoose, L. Shao, and R. G. Compton, "Cyclic voltammetry on electrode surfaces covered with porous layers: an analysis of electron transfer kinetics at singlewalled carbon nanotube modified electrodes," Sensors and Actuators, B, vol. 133, no. 2, pp. 462-466, 2008.

[30] G. P. Keeley and M. E. G. Lyons, "The effects of thin layer diffusion at glassy carbon electrodes modified with porous films of single-walled carbon nanotubes," International Journal of Electrochemical Science, vol. 4, no. 6, pp. 794-809, 2009.

[31] L. Xiao, G. G. Wildgoose, and R. G. Compton, "Exploring the origins of the apparent "electrocatalysis" observed at C60 filmmodified electrodes," Sensors and Actuators, B, vol. 138, no. 2, pp. 524-531, 2009.
[32] M. Ghalkhani and S. Shahrokhian, "Application of carbon nanoparticle/chitosan modified electrode for the square-wave adsorptive anodic striping voltammetric determination of Niclosamide," Electrochemistry Communications, vol. 12, no. 1, pp. 66-69, 2010.

[33] S. Shahrokhian and H. R. Zare-Mehrjardi, "Electrochemical synthesis of polypyrrole in the presence of congo red; application to selective voltammetric determination of dopamine in the presence of ascorbic acid," Electroanalysis, vol. 21, no. 2, pp. 157-164, 2009.

[34] M. C. Granger, J. Xu, J. W. Strojek, and G. M. Swain, "Polycrystalline diamond electrodes: basic properties and applications as amperometric detectors in flow injection analysis and liquid chromatography," Analytica Chimica Acta, vol. 397, no. 1-3, pp. 145-161, 1999. 


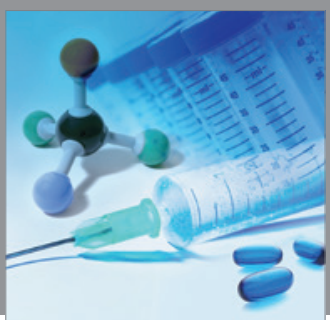

International Journal of

Medicinal Chemistry

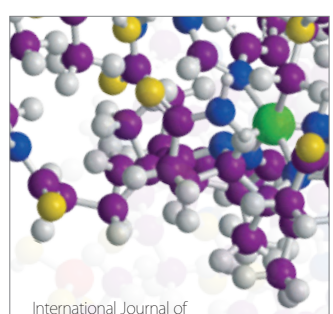

Carbohydrate Chemistry

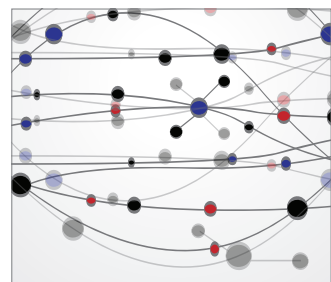

The Scientific World Journal
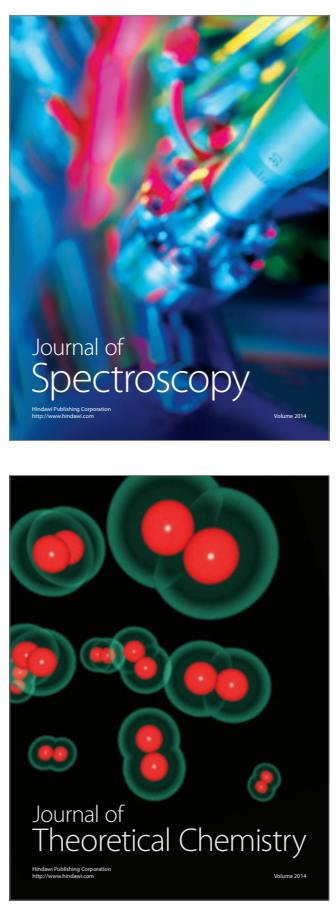
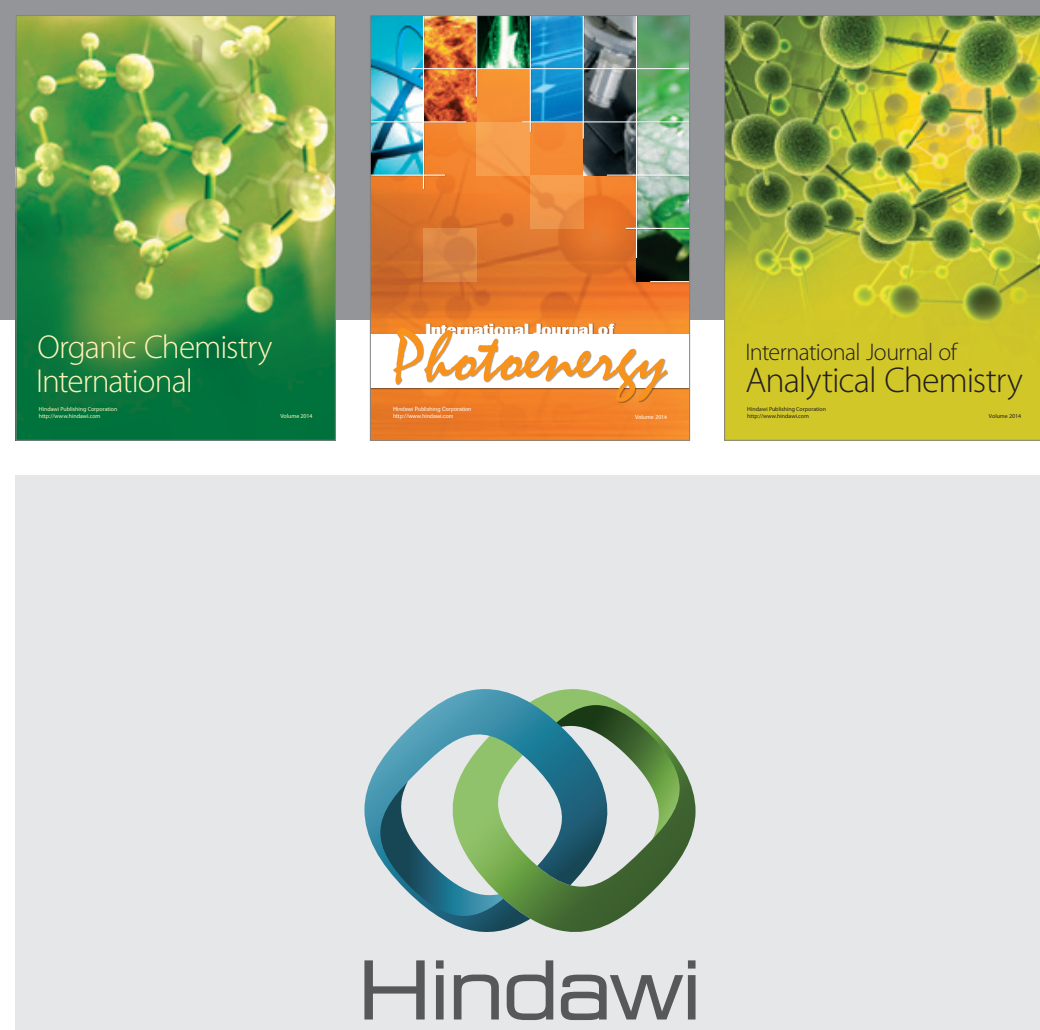

Submit your manuscripts at

http://www.hindawi.com
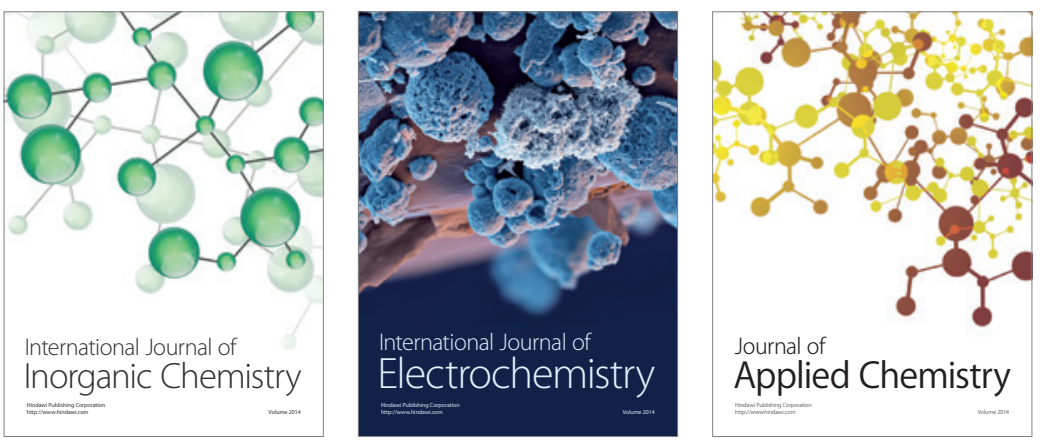

Journal of

Applied Chemistry
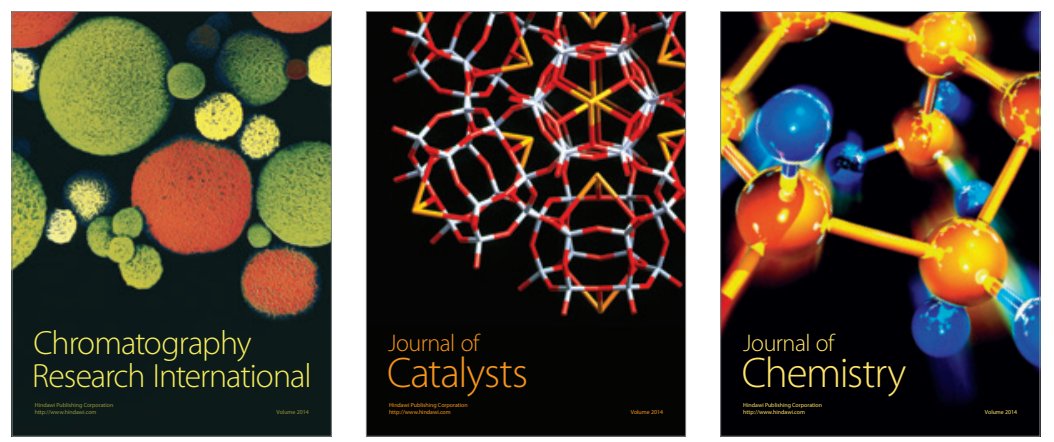
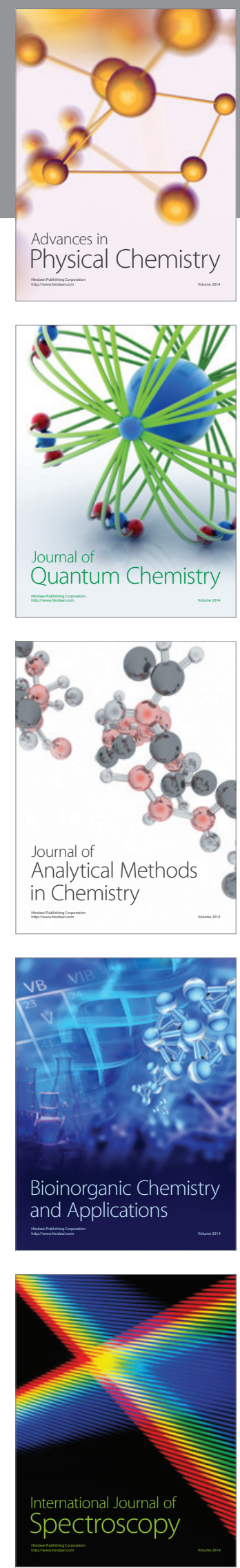